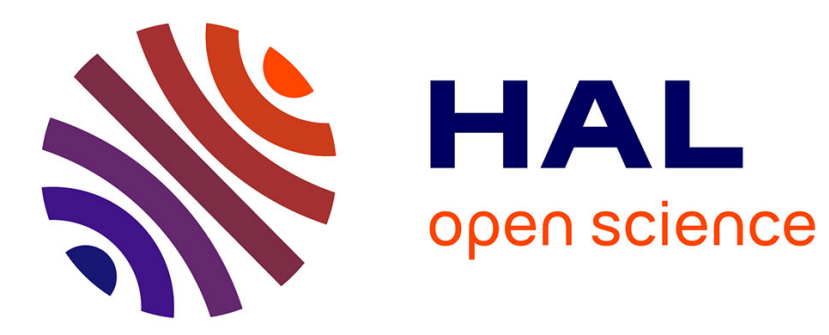

\title{
Divergence and flutter instabilities of some constrained two-degree-of-freedom systems
}

François Nicot, Jean Lerbet, Félix Darve

\section{To cite this version:}

François Nicot, Jean Lerbet, Félix Darve. Divergence and flutter instabilities of some constrained two-degree-of-freedom systems. Journal of Engineering Mechanics - ASCE, 2014, 140 (1), pp.47-52. 10.1061/(ASCE)EM.1943-7889.0000638 . hal-00787441

\section{HAL Id: hal-00787441 \\ https://hal.science/hal-00787441}

Submitted on 16 Jan 2020

HAL is a multi-disciplinary open access archive for the deposit and dissemination of scientific research documents, whether they are published or not. The documents may come from teaching and research institutions in France or abroad, or from public or private research centers.
L'archive ouverte pluridisciplinaire HAL, est destinée au dépôt et à la diffusion de documents scientifiques de niveau recherche, publiés ou non, émanant des établissements d'enseignement et de recherche français ou étrangers, des laboratoires publics ou privés. 


\title{
Divergence and Flutter Instabilities of Some Constrained Two-Degree-of-Freedom Systems
}

\author{
François Nicot ${ }^{1}$; Jean Lerbet ${ }^{2}$; and Félix Darve ${ }^{3}$
}

\begin{abstract}
It is now well known that a variety of instability modes can appear before the conventional plastic limit condition is met. In this note, both flutter and divergence instability modes are investigated. First, the criterion for detecting their occurrence is established, and the case of kinematically constrained discrete systems is investigated. Based on an illustrative example, the competition between the occurrences of each of these instability modes is analyzed, showing that the prevalence of a given mode is strongly related to both the loading conditions and the stiffness properties of the material system at hand.
\end{abstract}

Keywords: Bifurcation; Flutter instability; Divergence instability; Second-order work; Loading parameters; Failure; Structures.

\section{Introduction}

It is usual to distinguish between divergence instabilities and flutter instabilities. Roughly speaking, the first are linked to suddenly monotonously growing deformations, whereas the second correspond to cyclically increasing deformations (Bigoni 2000; Piccolroaz et al. 2006; Bigoni and Noselli 2011). In this paper, the authors will not return to these definitions and their related general theoretical framework, which can be found in several textbooks and seminal papers (Ziegler 1953; Bolotin 1963; Huseyin 1978; Leipholz 1970, 1987). Just keep in mind that both these instability modes can appear in discrete mechanical systems according to the loading conditions and the mass distributions. It is observed that, in some cases, divergence instability appears first, whereas in other cases, flutter instability occurs first. Indeed, very few contributions have been devoted to a comparison between these occurrences. In Lerbet et al. (2009), it was proved that if the second-order work criterion of stability (the stiffness matrix $\mathbf{K}$ is positive definite) is fulfilled, then all the coefficients of the characteristic polynomial $P(X)=\operatorname{det}(\mathbf{M} X+\mathbf{K})$ are strictly positive, where $\mathbf{M}$ is the mass matrix. This latter condition was shown to be one of the two conditions guaranteeing the flutter stability (Gallina 2003).

The purpose of this paper is thus to compare the occurrence of both these instability modes in general two-dimensional (2D) discrete systems. Because flutter instability is observed for dynamic conditions, the mass distribution plays an essential role in its development. For a comparison with divergence instability, it is thus necessary to consider a simple mass distribution, leading to a spherical mass matrix. This is the first basic assumption, which takes into account the fact that it is not possible to compare both these modes in a fully general manner.

\footnotetext{
${ }^{1}$ Professor, Institut de Recherche en Sciences et Technologies de l'Environnement (IRSTEA), ETNA-Geomechanics Group, Grenoble, 38402, France (corresponding author). E-mail: francois.nicot@cemagref.fr

${ }^{2}$ Professor, Univ. d'Evry Val d'Essonne, Unité de Formations et de Recherche en Sciences et Technologie, Evry, 91000, France.

${ }^{3}$ Professor, Laboratoire Sols Solides Structures, UJF-INPG-CNRS, Grenoble, 38041, France.
}

Then, it has been shown in a series of papers (Lerbet et al. 2012; Nicot et al. 2011a, 2012; Challamel et al. 2009, 2010) that, for nonconservative elastic discrete systems, some added kinematic constraints can modify the occurrence of divergence instability states, which can appear sooner (according to the loading parameter) than those related to the free system. The loss of positive definiteness for the stiffness matrix (the so-called second-order work criterion) has been shown to be the envelope of all the divergence instability curves if one takes into account all the possible kinematic constraints. The lowest boundary of the domain leading to divergence instabilities is thus constituted by the second-order work criterion.

As regards the 2D flutter instability, it has been proved (Kirillov 2007) that this instability mode can appear when the two eigenvalues of the stiffness matrix coincide. This condition will be used as the criterion for flutter instability to occur. In this framework, the criteria for both divergence and flutter instabilities can then be compared, and it is shown in this paper that, indeed, a clear analytical quantitative comparison can be performed. Finally, an illustration of the indicators obtained is presented in the case of airplane wings.

\section{Flutter against Divergence Instabilities}

\section{Introduction}

To illustrate the competition between flutter and divergence instabilities, a 2D undamped system is considered. See for instance Kounadis (1994, 1997, 2007), in which a thorough investigation of the occurrence of flutter instabilities in damped systems has been carried out.

In this context, any geometric configuration $\mathbf{X}=\left(X_{1}, X_{2}\right)$ of the system is defined by the variables $X_{1}$ and $X_{2}$, and its evolution over time is given by the fundamental equations

$$
\begin{aligned}
& M_{11} \ddot{X}_{1}+M_{12} \ddot{X}_{2}+K_{11} X_{1}+K_{12} X_{2}=0 \\
& M_{12} \ddot{X}_{1}+M_{22} \ddot{X}_{2}+K_{21} X_{1}+K_{22} X_{2}=0
\end{aligned}
$$

Governing Eqs. (1) can be transformed as follows:

$$
\ddot{Y}_{1}+H_{11} Y_{1}+H_{12} Y_{2}=0, \quad \ddot{Y}_{2}+H_{21} Y_{1}+H_{22} Y_{2}=0
$$

where $Y_{1}=S_{11} X_{1}+S_{12} X_{2} ; Y_{2}=S_{21} X_{1}+S_{22} X_{2} ; \mathbf{S}=$ square root of matrix $\mathbf{M}$; and $\mathbf{H}=(\mathbf{S})^{-1} \mathbf{K}(\mathbf{S})^{-1}$. Because the mass distribution of 
the system is fully embedded in the matrix $\mathbf{H}$, the spectral properties of $\mathbf{H}$ closely depend both on the stiffness properties and on the mass distribution of the system. On the contrary, the mechanism of divergence instability is related to the single stiffness matrix $\mathbf{K}$, omitting the mass distribution.

Henceforth, the contribution of the mass distribution will be disregarded in the investigation of the difference between the two instability modes induced by only the stiffness matrix. Therefore, the matrix $\mathbf{M}$ will be considered spherical, which implies for the matrix $\mathbf{S}$ that $S_{11}=S_{22}$ and $S_{12}=S_{21}=0$. Both matrices $\mathbf{H}$ and $\mathbf{K}$ are proportional and thereby have the same spectral properties. In this case, flutter instability analysis can be performed by considering the stiffness matrix $\mathbf{K}$. It should be pointed out that considering the mass distribution as uniform does not alter the generality of the subsequent analysis. If any (symmetric) mass matrix is considered, the flutter instability criterion should be discussed with the matrix $\mathbf{H}$. However, as $\mathbf{S}$ is symmetric, the natures of the quadratic forms associated with matrices $\mathbf{K}$ and $\mathbf{H}$ are the same. The divergence instability criterion can be thereby expressed by either $\mathbf{K}$ or $\mathbf{H}$. Thus, for a general mass distribution, all subsequent results can be preserved by replacing $\mathbf{K}$ with $\mathbf{H}$.

In what follows, it will be assumed that det $\mathbf{K}$ is not nil. (All eigenvalues of $\mathbf{K}$ are strictly positive.)

\section{Flutter Instability Analysis}

Flutter instability has been shown to occur as soon as the algebraic multiplicity of any eigenvalue is larger than its geometric multiplicity [see for example Ziegler (1953), Bolotin (1963), Leipholz (1970, 1987), and Huseyin (1978) for a more complete review of flutter instabilities]. In two dimensions, this situation occurs when the two eigenvalues coincide (Kirillov 2007).

The characteristic polynomial of $\mathbf{H}$ reads

$$
P_{H}(X)=X^{2}-\left(H_{11}+H_{22}\right) X+H_{11} H_{22}-H_{12} H_{21}
$$

It admits a double root as soon as the discriminant is vanishing, which gives

$$
\left(H_{11}+H_{22}\right)^{2}-4\left(H_{11} H_{22}-H_{12} H_{21}\right)=0
$$

By taking advantage of the proportionality between both $\mathbf{H}$ and $\mathbf{K}$ and after rearranging the terms, Eq. (4) also reads

$$
\left(K_{11}-K_{22}\right)^{2}+4 K_{12} K_{21}=0
$$

Eq. (5) stands as the criterion for the occurrence of flutter instability for the specific 2D system considered in this paper. Flutter instabilities occur as soon as $I_{f}=0$ with $I_{f}=\left(K_{11}-K_{22}\right)^{2}+4 K_{12} K_{21}$.

Moreover, the differential system in Eq. (2) can be solved analytically in a direct way. As det $\mathbf{K} \neq 0$, the same holds for $\mathbf{H}$, and det $\mathbf{H} \neq 0$. Thus, $H_{12}$ and $H_{22}$ cannot be equal to zero simultaneously. Assume that $H_{12} \neq 0$. Then, Eq. (2) can be rewritten as

$$
\begin{aligned}
& Y_{1}^{(4)}+\left(H_{11}+H_{22}\right) \ddot{Y}_{1}+\left(H_{11} H_{22}-H_{12} H_{21}\right) Y_{1}=0 \\
& Y_{2}=-\left(\ddot{Y}_{1}+H_{11} Y_{1}\right) / H_{12}
\end{aligned}
$$

The analytical form of the solution of the first differential equation depends on the sign of the discriminant $\Delta_{H}=\left(H_{11}+H_{22}\right)^{2}$ $-4\left(H_{11} H_{22}-H_{12} H_{21}\right)$ or $\Delta_{K}=\left(K_{11}-K_{22}\right)^{2}+4 K_{12} K_{21}$. Flutter instability occurs when $\Delta_{K}=0$. In this case, the component $Y_{1}(t)$ can be shown to be a linear combination of terms $(\alpha+\beta t) \exp (r t)$ where $r^{2}=-\left(H_{11}+H_{22}\right) / 2$. The amplitude of the component increases over time.

\section{Divergence Instability Analysis}

Divergence instability will be investigated in the case where the following additional kinematic constraint is prescribed for the system:

$$
a_{1} X_{1}+a_{2} X_{2}=0
$$

Both Eqs. (1) and (7) can be merged into the following system by introducing a Lagrangian parameter $\lambda$ :

$$
\begin{aligned}
M_{11} \ddot{X}_{1}+K_{11} X_{1}+K_{12} X_{2}+\lambda a_{1} & =0, \\
M_{22} \ddot{X}_{2}+K_{21} X_{1}+K_{22} X_{2}+\lambda a_{2} & =0, \\
a_{1} X_{1}+a_{2} X_{2} & =0
\end{aligned}
$$

Eliminating the parameter $\lambda$ between the first two Eqs. (8) gives

$$
\begin{aligned}
& M_{11} a_{2} \ddot{X}_{1}-M_{22} a_{1} \ddot{X}_{2}+\left(K_{11} a_{2}-K_{21} a_{1}\right) X_{1} \\
& \quad+\left(K_{12} a_{2}-K_{22} a_{1}\right) X_{2}=0
\end{aligned}
$$

Combined with the third Eq. (8), Eq. (9) can be expressed as

$$
\begin{aligned}
& \left(M_{11} a_{2}^{2}+M_{22} a_{1}^{2}\right) \ddot{X}_{1}+\left[K_{11} a_{2}^{2}-\left(K_{12}+K_{21}\right) a_{1} a_{2}\right. \\
& \left.\quad+K_{22} a_{1}^{2}\right] X_{1}=0
\end{aligned}
$$

Noting that

$$
M_{11} a_{2}^{2}+M_{22} a_{1}^{2}={ }^{t}\left(a_{2}-a_{1}\right) \mathbf{M}\left(a_{2}-a_{1}\right)
$$

and

$$
K_{11} a_{2}^{2}-\left(K_{12}+K_{21}\right) a_{1} a_{2}+K_{22} a_{1}^{2}={ }^{t}\left(a_{2}-a_{1}\right) \mathbf{K}^{s}\left(a_{2}-a_{1}\right)
$$

finally yields

$$
\begin{aligned}
& { }^{t}\left(a_{2}-a_{1}\right) \mathbf{M}\left(a_{2}-a_{1}\right) \ddot{X}_{1}+{ }^{t}\left(a_{2}-a_{1}\right) \mathbf{K}^{s}\left(a_{2}-a_{1}\right) X_{1}=0, \\
& X_{2}=-\frac{a_{1}}{a_{2}} X_{1}
\end{aligned}
$$

Eq. (13) stands as the system's governing equation, describing how the geometric configuration $\mathbf{X}=\left(X_{1}, X_{2}\right)$ evolves over time from the initial state $\mathbf{X}_{o}=\left(X_{1 o}, X_{2 o}\right)=(0,0)$ when for example a velocity disturbance $\dot{\mathbf{X}}_{o}=\left(\dot{X}_{1 o}, \dot{X}_{2 o}\right)$ is applied. In this case, it is worth noting that the coordinates $\dot{X}_{1 o}$ and $\dot{X}_{2 o}$ have to meet the kinematic constraint from Eq. (7), so that both vectors $\dot{\mathbf{X}}_{o}$ and $\mathbf{A}=\left(a_{2},-a_{1}\right)$ are collinear.

As the mass term ${ }^{t}\left(a_{2},-a_{1}\right) \mathbf{M}\left(a_{2},-a_{1}\right)$ is always strictly positive, the nature of the dynamical response of the system depends only on the sign of the quantity ${ }^{t}\left(a_{2},-a_{1}\right) \mathbf{K}^{s}\left(a_{2},-a_{1}\right)$. When $\mathbf{K}^{s}$ admits at least one negative eigenvalue, then the vector $\mathbf{A}=\left(a_{2},-a_{1}\right)$ exists, so that the quantity ${ }^{t}\left(a_{2},-a_{1}\right) \mathbf{K}^{s}\left(a_{2},-a_{1}\right)$ is negative.

The occurrence of a divergence instability mode is related to the spectral properties of the quantity $\mathbf{K}^{s}$ together with the application of a suitable kinematic constraint. Assuming that all eigenvalues of $\mathbf{K}^{s}$ are initially strictly positive, divergence instability is expected once the determinant of $\mathbf{K}^{s}$ has first vanished, which reads as $I_{d}=0$ with $I_{d}=\left(K_{12}+K_{21}\right)^{2}-4 K_{11} K_{22}$. 


\section{Discussion}

From the previous considerations, the following main results can be put forward:

- A flutter instability mode occurs as soon as the quantity $I_{f}$ $=\left(K_{11}-K_{22}\right)^{2}+4 K_{12} K_{21}$ vanishes and takes negative values.

- A divergence instability mode is expected as soon as the quantity $I_{d}=\left(K_{12}+K_{21}\right)^{2}-4 K_{11} K_{22}$ vanishes and takes negative values. It is worth introducing the function $f(x, y, z, t)=(x-y)^{2}+4 z t$. Indeed, it can be noted that $I_{f}=f\left(K_{11}, K_{22}, K_{12}, K_{21}\right)$ and $I_{d}=-f\left(K_{12},-K_{21}, K_{11},-K_{22}\right)$.

Thus, $\left(K_{11}, K_{22}\right)$ and $\left(K_{12},-K_{21}\right)$ play a symmetric role in both criteria. As a consequence, some common properties can be inferred.

First and for a strictly mathematical point of view, if a stiffness operator

$$
\mathbf{K}=\left[\begin{array}{ll}
K_{11} & K_{12} \\
K_{21} & K_{22}
\end{array}\right]
$$

exists that satisfies $I_{f}=0$, then the stiffness operator

$$
\tilde{\mathbf{K}}=\left[\begin{array}{cc}
K_{12} & K_{11} \\
-K_{22} & -K_{21}
\end{array}\right]
$$

satisfies $I_{d}=0$. Furthermore, by denoting $\Delta=K_{11} K_{22}-K_{12} K_{21}$ as the determinant of the stiffness operator $\mathbf{K}$, the following relations can be obtained:

$$
\begin{aligned}
& I_{f}=\left(K_{11}+K_{22}\right)^{2}-4 \Delta \\
& I_{d}=\left(K_{12}-K_{21}\right)^{2}-4 \Delta
\end{aligned}
$$

If flutter instabilities have occurred, then $I_{f}=0$, which yields that $\Delta \geq 0$. Likewise, if divergence instabilities have occurred, then $I_{d}=0$, which yields that $\Delta \geq 0$. Thus, both instability modes can appear before the conventional failure criterion $\Delta=0$ is met. More specifically, if one of those instability modes should appear, then it appears necessarily before the conventional failure criterion $\Delta=0$ is met.

Even though $\mathbf{K}^{s}$ admits negative eigenvalues, no divergence instability takes place in the absence of a suitable kinematic constraint. In that case, flutter instabilities prevail. This is the only instability mode that can be observed before the conventional failure condition $\Delta=0$ is met.

If a suitable kinematic constraint is added, then divergence instability will occur if flutter instability has not yet appeared. The occurrence of the first instability mode, among flutter and instability modes, depends on the stiffness properties of the system. Depending upon the loading, the terms $K_{i j}$ of the stiffness operator evolve. As this evolution is closely related to the system (in particular, its geometry), no general conclusion can be drawn about which mode will first appear.

However, it should be emphasized that flutter instabilities appear as a natural instability mode in the sense that no specific constraint is required, whereas a suitable kinematic constraint is required to observe divergence instabilities. Basically, the kinematic constraint enables the differential equations governing the dynamic evolution of the system to be uncoupled, giving rise to a one-dimensional equation. The divergence of this one-dimensional differential equation is controlled by the sign of the second-order work along the direction defined by the kinematic constraint $\left[\operatorname{term}^{t}\left(a_{2},-a_{1}\right) \mathbf{K}^{s}\left(a_{2},-a_{1}\right)\right]$.

As an illustration, the case of airplane wings with aeroelastic effects is investigated in the section "Instability Modes of Airplane Wings with Aeroelastic Effects."

\section{Instability Modes of Airplane Wings with Aeroelastic Effects}

\section{Physical Model}

It should be emphasized that the 2D model of an airplane with aeroelastic effects has been extensively investigated in the past [see for instance Bolotin (1963) for a nonlinear analysis including damping; more recently, a complete review without kinematic constraints can be found in Seyranian and Kirillov (2001) and Kirillov and Seyranian (2002)].

A rigid plate of unit width and specific mass $\mu$ per unit length is considered throughout this section. The plate is suspended on springs of stiffness $C_{1}$ and $C_{2}$ as shown in Fig. 1. Initially in a horizontal position of static equilibrium, the plate is loaded by wind of velocity $v$, characterized by the wind force resultant $F=\xi v^{2} \theta$ acting at a distance $a$ ahead of the downwind end of the plate, where $\xi$ is a constant parameter and $\theta$ is the rotation of the plate. The foregoing definition of $F$ is valid only for very slow oscillations. The location of the resultant of the aerodynamic forces on the plate is called the aerodynamic center in aeroelasticity. For 2D incompressible flow, this center is located at $a=3 b / 4$, whereas for supersonic flow, it is located at $a=b / 2$ (Bažant and Cedolin 2003).

If the deflection from the static equilibrium position at the midpoint is denoted as $w$, the fundamental equations of vertical forces and moments around the center of the plate are written as

$$
\mathbf{M}\left[\begin{array}{c}
\ddot{w} \\
\ddot{\theta}
\end{array}\right]+\mathbf{K}\left[\begin{array}{c}
w \\
\theta
\end{array}\right]=\mathbf{0}
$$

with

$$
\mathbf{M}=\left[\begin{array}{cc}
\mu b & 0 \\
0 & \mu b^{3} / 12
\end{array}\right]
$$

and

$$
\mathbf{K}=\left[\begin{array}{ll}
K_{11} & K_{12} \\
K_{21} & K_{22}
\end{array}\right]
$$

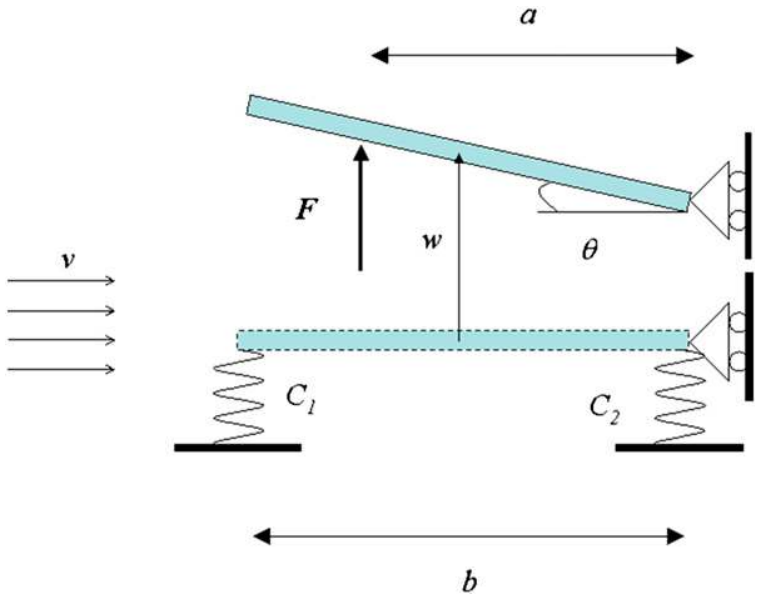

Fig. 1. A 2D model for airplane wings under aerodynamic forces for a free system 
The stiffness matrix is detailed as follows:

$$
\begin{aligned}
& K_{11}=C_{1}+C_{2}, \quad K_{12}=b \frac{C_{1}-C_{2}}{2}-\xi v^{2}, \quad K_{21}=b \frac{C_{1}-C_{2}}{2}, \\
& K_{22}=\frac{b^{2}}{4}\left(C_{1}+C_{2}\right)-\xi v^{2}\left(a-\frac{b}{2}\right)
\end{aligned}
$$

After a normalization procedure for the mass matrix, the differential equations can also be presented in an equivalent way

$$
\left[\begin{array}{c}
\ddot{w} \\
b \ddot{\theta}
\end{array}\right]+\frac{C_{1}+C_{2}}{\mu b} \tilde{\mathbf{K}}\left[\begin{array}{c}
w \\
b \theta
\end{array}\right]=\mathbf{0}
$$

where the modified stiffness matrix is now given by

$$
\tilde{\mathbf{K}}=\left[\begin{array}{cc}
1 & \frac{c}{2}-\chi \\
6 c & 3-12 \chi\left(\frac{a}{b}-\frac{1}{2}\right)
\end{array}\right]
$$

with $c=\left(C_{1}-C_{2}\right) /\left(C_{1}+C_{2}\right)$ and $\chi=\xi v^{2} /\left[b\left(C_{1}+C_{2}\right)\right]$.

\section{Numerical Investigation of Flutter/Divergence Instability Occurrence}

The occurrence of flutter and divergence instability modes can be investigated according to the stiffness operator given in Eq. (19). In what follows, the vanishing of terms $I_{f}$ and $I_{d}$ is analyzed for different values of $c$ (ranging between -1 and 1 ) and for $a / b$ equal to $1 / 2$ or $3 / 4$. The parameter $c$ accounts for the stiffness properties of

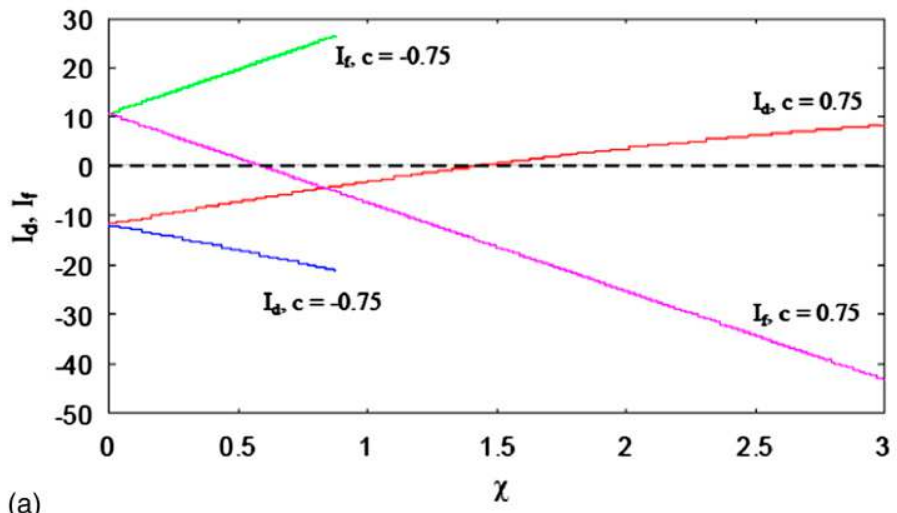

(a)

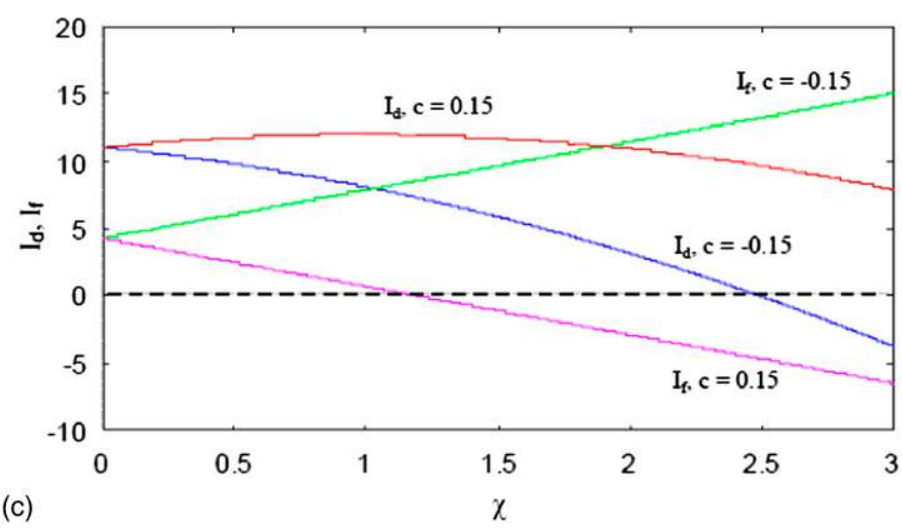

the system (distribution of stiffness between the two springs), whereas the parameter $a / b$ relates to the loading. The evolution of terms $I_{f}$ and $I_{d}$ over the loading parameter $\chi$ is reported in Figs. 2 and 3. Moreover, as the occurrence of those instability modes is expected before the divergence of the free system occurs, the curves are plotted for the values of $\chi$ that ensure that det $\tilde{\mathbf{K}}$ is strictly positive. (All eigenvalues of $\tilde{\mathbf{K}}$ are strictly positive.)

In the supersonic-flow regime $(a / b=1 / 2)$, divergence instabilities always precede flutter instabilities for large values of $c$. When $c$ decreases $(|c|=0.5)$, divergence instabilities always precede flutter instabilities for negative values of $c$, whereas the reverse holds for positive values of $c$. It should be noted that flutter instabilities never occur when $c$ is negative or nil (Fig. 2).

The same conclusion can be drawn in the $2 \mathrm{D}$ incompressibleflow regime $(a / b=3 / 4)$. As seen in Fig. 3, divergence instabilities always precede flutter instabilities for large values of $c$ (until $c=0.4)$. When $c$ decreases $(|c|=0.15)$, divergence instabilities precede flutter instabilities for negative values of $c$, whereas the reverse holds for positive values of $c$. In the particular case of $c=0$, $I_{f}$ vanishes (minimum value) at $\chi=2 / 3$, and $I_{d}$ vanishes at $\chi=4 \sqrt{3}-6$; flutter instabilities precede divergence instabilities.

It is worth stressing again that the divergence instability occurrence requires that a suitable kinematic constraint is prescribed. The free system depicted in Fig. 1 cannot be affected by a divergence instability before the determinant of $\tilde{\mathbf{K}}$ has vanished.

Divergence instability can be observed with the constrained system as shown in Fig. 4. This mechanism models the connection of the wing to the aircraft body. The two kinematic parameters are linked by a mechanism parameterized by a fixed polar value $x_{0}$ as follows:

$$
\tan \theta=\frac{w}{x_{0}+b / 2}
$$
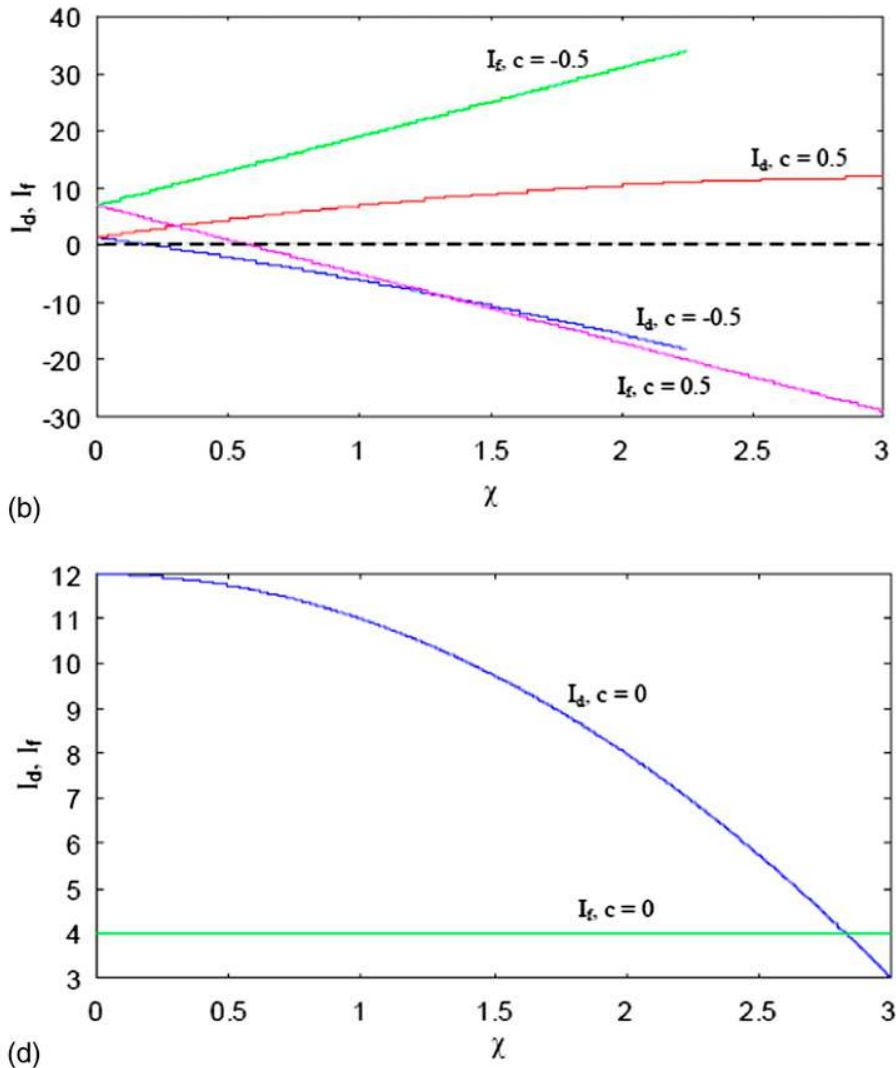

Fig. 2. Divergence against flutter instability modes for $a / b=0.5$ 


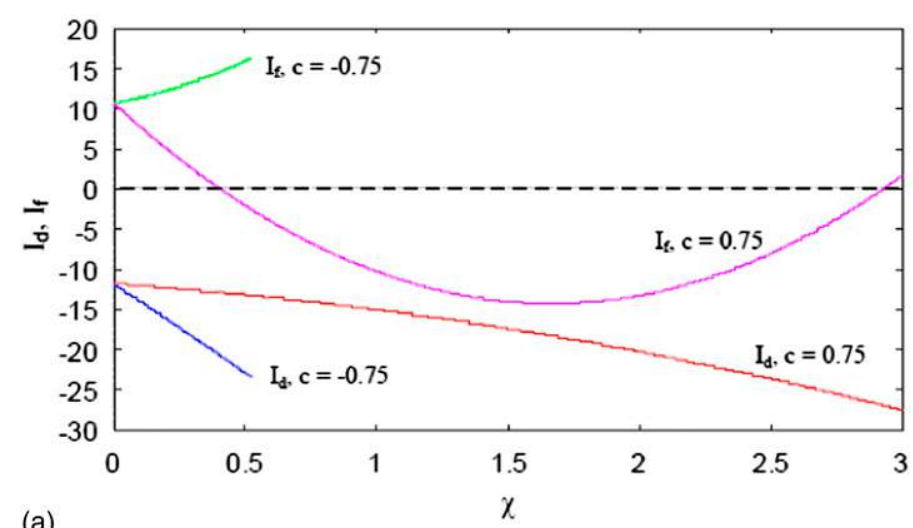

(a)

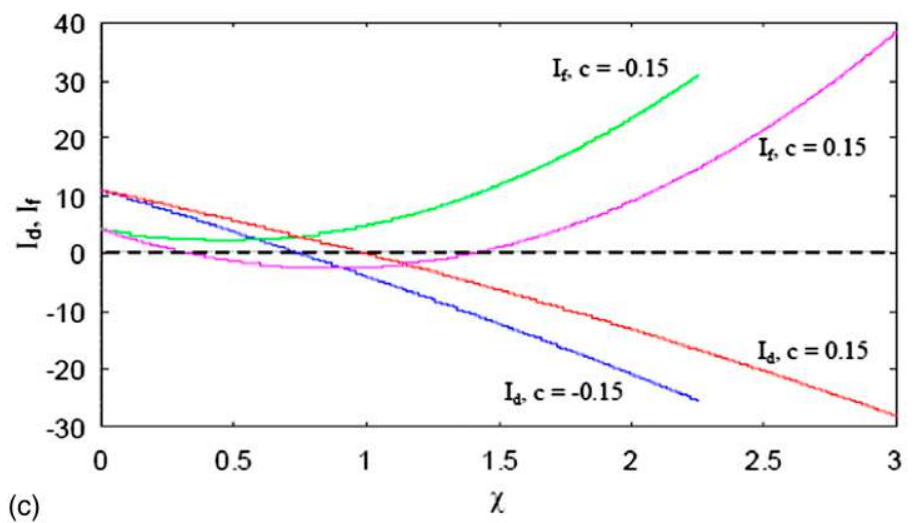

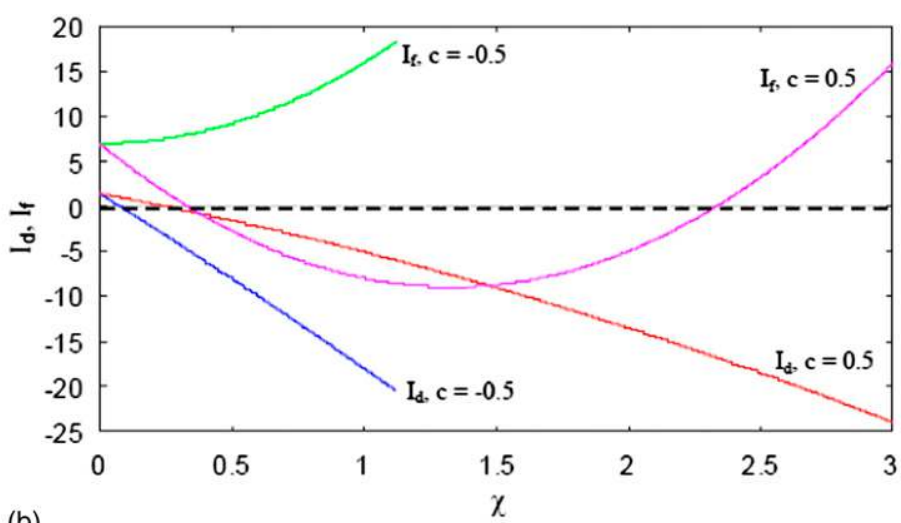

(b)

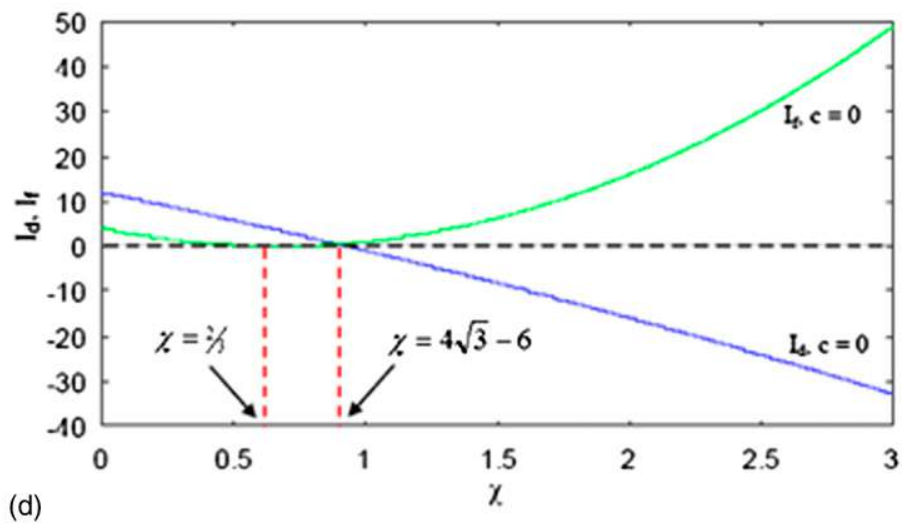

(d)

Fig. 3. Divergence against flutter instability modes for $a / b=0.75$

If the analysis is limited to small values $\theta$, Eq. (20) can be linearized, which gives

$$
w-\theta\left(x_{0}+\frac{b}{2}\right)=0
$$

Eq. (21) corresponds to a linear kinematic constraint $\alpha_{1} w+\alpha_{2} \theta b$ $=0$ of the same form as that given in Eq. (7). When the determinant of $\tilde{\mathbf{K}}^{s}$ vanishes, divergence instability appears if the direction of the kinematic constraint from Eq. (21) belongs to the negative isotropic cone of $\tilde{\mathbf{K}}^{s}$. (This cone gathers all vectors $\mathbf{x}$ that ensure that the quadratic form associated with $\tilde{\mathbf{K}}^{s}$ is negative: $\left.\tilde{K}_{i j}^{s} x_{i} x_{j}<0\right)$. This issue has been thoroughly investigated in Nicot et al. (2011b, 2012).

Finally, this investigation points out how the prevalence of the divergence or flutter instability mode closely depends upon the stiffness, geometry, and loading properties. This example clearly emphasizes that no general rule can be inferred.

This analysis was carried out in the specific case where the mass distribution is homogeneous. In this context, both flutter and divergence analyses were conducted by considering the stiffness operator, excluding mass terms. In more general situations, the flutter instability analysis has to account for mass distribution through a mixed operator $\mathbf{H}=\mathbf{S}^{-1} \mathbf{K} \mathbf{S}^{-1}$. On the other hand, the divergence instability analysis is carried out through the pure stiffness operator $\mathbf{K}$ (symmetric part). This intricacy heavily prevents a general analysis of the competition between divergence and flutter instability modes.

\section{Concluding Remarks}

This note has investigated the competition between both flutter and divergence instability modes applied to the context of

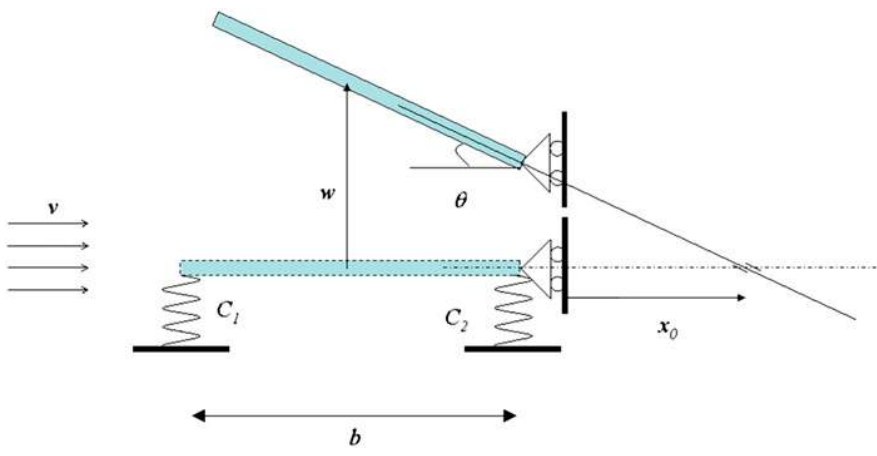

Fig. 4. A $2 \mathrm{D}$ model for airplane wings under aerodynamic forces for a constrained system

two-degree-of-freedom systems. Based on the linearized system of the balance equations, the asymptotic behavior of the system under the effect of initial velocity disturbances is analyzed. In particular, the case of constrained systems subjected to a set of holonomic kinematic constraints is considered. The following results should be put forward:

- In the particular case of a uniform mass distribution, both divergence and flutter instability criteria have been formulated in a symmetric way. (Diagonal and out-of-diagonal terms of the stiffness matrix play symmetric roles.)

- Both divergence and flutter instability modes can appear before the conventional plastic failure condition is met (occurrence of undefined strains under a constant stress, which requires that the stiffness matrix $\mathbf{K}$ be singular). If one of those instability modes 
appears, this is necessarily before the conventional plastic failure condition is met.

- Even though the symmetric part of the stiffness operator admits negative eigenvalues, no divergence instability takes place in the absence of a suitable kinematic constraint. In that case, flutter instabilities prevail. This is the only instability mode that can be observed before the conventional plastic failure condition is met.

- If a suitable kinematic constraint is added, then divergence instability will occur if flutter instability has not yet appeared. The occurrence of the first instability mode, among flutter and instability modes, depends both on the stiffness properties of the system (including geometric effects) and on the loading. Hence, no general conclusion can be drawn about which mode will first appear.

- Flutter instabilities appear as a natural instability mode in the sense that no specific constraint is required, whereas a suitable kinematic constraint is required to observe divergence instabilities.

\section{Acknowledgments}

The authors express their gratitude to the French Research Network MeGe (Multiscale and multi-physics couplings in geoenvironmental mechanics, GDR CNRS 3176, 2008-2011) for having supported this work.

\section{References}

Bažant, Z., and Cedolin, L. (2003). Stability of structures, Dover, Mineola, NY.

Bigoni, D. (2000). "Bifurcation and instability of non associative elastic plastic solids." Chapter 1, Material instabilities in elastic and plastic solids, H. Petrik, ed., Springer, New York, 1-52.

Bigoni, D., and Noselli, G. (2011). "Experimental evidence of flutter and divergence instabilities induced by dry friction." J. Mech. Phys. Solids, 59(10), 2208-2226.

Bolotin, V. V. (1963). Nonconservative problems of the theory of elastic stability, Macmillan, New York.

Challamel, N., Nicot, F., Lerbet, J., and Darve, F. (2009). "On the stability of non-conservative elastic systems under mixed perturbations." Eur. J. Environ. Civ. Eng., 13(3), 347-367.
Challamel, N., Nicot, F., Lerbet, J., and Darve, F. (2010). "Stability of nonconservative elastic structures under additional kinematics constraints." Eng. Struct., 32(10), 3086-3092.

Gallina, P. (2003). "About the stability of non-conservative undamped systems." J. Sound Vibrat., 262(4), 977-988.

Huseyin, K. (1978). Vibrations and stability of multiple parameter systems, Kluwer, Dordrecht, Netherlands.

Kirillov, O. N. (2007). "Destabilization paradox due to breaking the Hamiltonian and reversible symmetry." Int. J. Non-linear Mech., 42(1), 71-87.

Kirillov, O. N., and Seyranian, A. P. (2002). "Metamorphoses of characteristic curves in circulatory systems." J. Appl. Math. Mech., 66(3), 371-385.

Kounadis, A. N. (1994). "On the failure of static stability analyses of nonconservative systems in regions of divergence instability." Int. J. Solids Struct., 31(15), 2099-2120.

Kounadis, A. N. (1997). "Non-potential dissipative systems exhibiting periodic attractors in region of divergence." Chaos Solitons Fractals, $8(4), 583-612$.

Kounadis, A. N. (2007). "Flutter instability and other singularity phenomena in symmetric systems via combination of mass distribution and weak damping." Int. J. Non Linear Mech., 42(1), 24-35.

Leipholz, H. H. E. (1970). Stability theory, Academic Press, New York.

Leipholz, H. H. E. (1987). Stability theory: An introduction to the stability of dynamic systems and rigid bodies, 2nd Ed., Wiley, New York.

Lerbet, J., Absi, E., and Rigolot, A. (2009). "About the stability of nonconservative undamped elastic systems: Some new elements." Int. J. Struct. Stab. Dyn., 9(2), 357-367.

Lerbet, J., Aldowaji, M., Challamel, N., Nicot, F., Prunier, F., and Darve, F. (2012). "P-positive definite matrices and stability of nonconservative systems." Z. Angew. Math. Mech., 92(5), 409-422.

Nicot, F., Challamel, N., Lerbet, J., Prunier, F., and Darve, F. (2011a). "Bifurcation and generalized mixed loading conditions in geomaterials." Int. J. Numer. Anal. Methods Geomech., 35(13), 1409-1431.

Nicot, F., Challamel, N., Lerbet, J., Prunier, F., and Darve, F. (2012). "Some insights into structure instability and the second-order work criterion." Int. J. Solids Struct., 49(1), 132-142.

Nicot, F., Hadda, N., Bourrier, F., Sibille, L., and Darve, F. (2011b). "Failure mechanisms in granular media, a discrete element analysis." Granul. Matter, 13(3), 255-260.

Piccolroaz, A., Bigoni, D., and Willis, J. R. (2006). "A dynamical interpretation of flutter instability in a continuous medium." J. Mech. Phys. Solids, 54(11), 2391-2417.

Seyranian, A. P., and Kirillov, O. N. (2001). "Bifurcation diagrams and stability boundaries of circulatory systems." Theor. Appl. Mech., 26, 135-138.

Ziegler, H. (1953). "Linear elastic stability. A critical analysis of methods." Z. Angew. Math. Phys., 4(2), 89-121. 